\title{
Comparison of vascular access outcomes in patients with end-stage renal disease attributed to systemic lupus erythematosus vs. other causes: a retrospective cohort study
}

Laura C. Plantinga ${ }^{1 *}$, S. Sam Lim², Rachel E. Patzer ${ }^{3,4}$, Stephen O. Pastan ${ }^{1,4}$ and Cristina Drenkard²

\begin{abstract}
Background: U.S. hemodialysis patients with systemic lupus erythematosus (SLE) and end-stage renal disease (ESRD) are less likely than other ESRD patients to have a permanent vascular access (fistula or graft) in place at the dialysis start. We examined whether vascular access outcomes after dialysis start differed for SLE vs. other ESRD patients.
\end{abstract}

Methods: Among U.S. patients initiating hemodialysis in 2010 with only a catheter ( $n=40,911 ; 384$ with SLE) and using a permanent access on first dialysis ( $n=13,073 ; 48$ with SLE), we examined the association of SLE status with time to first placement of a permanent access (among catheter-only patients) and to loss of access patency (among patients using a permanent access on first dialysis), both censored 1 year after dialysis start, using multivariable Cox proportional hazards models.

Results: Among catheter-only patients, $46.1 \%$ vs. $54.5 \%$ of those with SLE-ESRD vs. other ESRD had a permanent access placed within 1 year after dialysis start. However, with adjustment, there was no association of 1-year placement with SLE status [HR $=1.00(95 \% \mathrm{Cl}, 0.86-1.17)]$. SLE-ESRD vs. other ESRD patients starting dialysis with a permanent access were less likely to experience a 1-year loss of patency (43.8 \% vs. $55.0 \%$ ), but this association was not statistically significant after adjustment $[H R=0.88(0.57-1.37)]$.

Conclusion: These results suggest that SLE-ESRD patients starting dialysis with a catheter are not more likely to have a permanent access placed in the first year of dialysis, despite an observed lack of association of SLE status with subsequent loss of vascular access patency among those starting dialysis with a permanent access.

\section{Background}

End-stage renal disease (ESRD) patients who undergo hemodialysis with a permanent vascular access [arteriovenous fistula (AVF) or grafts (AVG)] rather than a temporary catheter generally have better outcomes [1-7] and lower associated healthcare costs [8]. Thus, national U.S. clinical guidelines promote the early placement of AVFs and AVG (at least 6 months and 3-6 weeks prior

\footnotetext{
*Correspondence: laura.plantinga@emory.edu

${ }^{1}$ Division of Renal Medicine, Department of Medicine, Emory University, 101 Woodruff Circle, 5105 Woodruff Memorial Building, Atlanta, GA 30322, USA

Full list of author information is available at the end of the article
}

to anticipated dialysis start, respectively), such that a mature, functioning vascular access is in place on first dialysis [9]. Centers for Medicare \& Medicaid Services (CMS) tracks early placement through the ESRD Medical Evidence form (CMS-2728), which collects data on access type at first dialysis for all patients starting ESRD treatment. CMS also promotes the placement of a permanent vascular access among hemodialysis patients through regionally implemented quality incentive programs such as Fistula First, Catheter Last [10].

Previously, we found that systemic lupus erythematosus (SLE) patients who developed ESRD were $40 \%$ less 
likely to have a permanent vascular access in place at the start of dialysis than other ESRD patients, despite being more likely than other ESRD patients to have other indicators of advanced ESRD care planning, such as receipt of pre-ESRD nephrology care and earlier placement on the deceased donor kidney transplant waitlist $[11,12]$. Further, O'Shaughnessy et al. [13] recently noted that ESRD patients with SLE were also less likely than ESRD patients with other types of glomerulonephritis to have an AVF in place at dialysis start. Here, using national claims data on ESRD patients, we examined whether subsequent placement of permanent vascular access differed for SLE-ESRD patients compared to other ESRD patients who started hemodialysis with only a catheter. Additionally, among U.S. patients who used a permanent access on their first hemodialysis treatment, we examined whether early loss of patency, as evidenced by procedures aimed at maintaining vascular access patency or placement of a new vascular access, differed for SLE-ESRD vs. other ESRD patients.

\section{Methods}

\section{Study population and data sources}

For this retrospective cohort study, data from the CMS2728 and Part A (inpatient) and Part B (outpatient) claims were obtained from the United States Renal Data System (USRDS) [14]. The study was approved by the Emory Institutional Review Board. Analyses were limited to patients starting dialysis in 2010, the most recent year available such that all patients had at least a year of potential follow-up. A total of 117,836 incident adult and pediatric ESRD patients were identified who initiated treatment from $1 / 1 / 10$ to $12 /$ $31 / 10$. Patients were excluded if they had a missing attributed cause of ESRD $[n=2,190(1.9 \%)]$; did not start on hemodialysis $[n=19,646$ (17.0\%); had fewer than 90 days of hemodialysis [ $n=4,683(4.9 \%)$ ], did not have information on starting vascular access $[n=810(0.9 \%)]$, or did not have evidence of Part B claims [ $n=23,381$ (25.8 \%)], leaving 67,478 patients (517 with SLE-ESRD and 66,961 with other ESRD; Fig. 1). Patients without Part B claims were excluded to avoid differential ascertainment of outpatient vascular

U.S. patients starting ESRD treatment $1 / 1 / 10-12 / 31 / 10$

$n=117,836$

$\downarrow$-2190 with missing attributed ESRD cause

Patients with known attributed cause of ESRD

$n=115,646$

1,020SLE/114,6260ther

$-19,646$ not starting on hemodialysis

Hemodialysis patients

$n=96,000$

812 SLE/95,188 Other

$\downarrow-4,683$ with $<90$ days of follow-up

$\geq 90$ days of follow-up

$n=91,317$

782 SLE/90,535 Other

$\downarrow-810$ without information on starting access

With starting access information

90,507

777SLE/89,730 Other

\rfloor$-23,381$ without Part B/outpatient claims data

With Part B/outpatient claims data

67,478

517SLE/66,961 Other

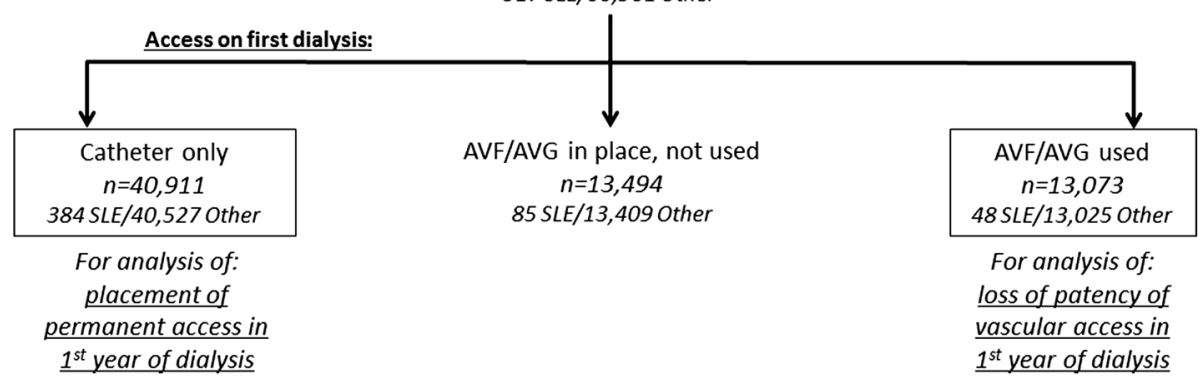

Fig. 1 Selection of study population among U.S. 2010 incident dialysis patients, overall and by systemic lupus erythematosus-attributed and other end-stage renal disease. AVF, arteriovenous fistula; AVG, arteriovenous graft; ESRD, end-stage renal disease; SLE, systemic lupus erythematosus 
access events among those who opted out of Part B coverage.

\section{Study variables \\ SLE status}

SLE-ESRD was defined as ESRD attributed to CMS-2728

ICD-9 code 710.0 (SLE). Other ESRD (the referent group) included all other non-missing ICD-9 codes listed on the CMS-2728.

\section{Vascular access at dialysis start}

Vascular access at dialysis start was determined from the CMS-2728. Vascular access at dialysis start was categorized as: having a catheter only [no permanent access (AVF or AVG) in place], having a permanent access in place but not used on first dialysis, and having a permanent access that was used on first dialysis.

\section{Vascular access placement in first year of dialysis}

Placement of a permanent access (AVF or AVG) was determined from Parts A and B claims data using Healthcare Common Procedure Coding System (HCPCS) codes (Additional file 1: Table S1). The date of placement was defined as the first date after start of dialysis in which an inpatient or outpatient code for placement was used.

\section{Loss of vascular access patency in first year of dialysis}

Loss of patency of a permanent access (AVF or AVG) was similarly determined from Part A and B claims data using HCPCS codes for revision procedures on the starting access or placement of new accesses (AVF, AVG, or catheter; Additional file 1: Table S1). The date of loss of patency was defined as the first date after start of dialysis on which an inpatient or outpatient code for a revision or placement was used.

\section{Other variables}

Incident age, sex, and treatment modalities were obtained from the USRDS standard analytic files. Race/ethnicity, primary insurance at start of ESRD, smoking status, body mass index (BMI), and presence of comorbid conditions were all obtained from the CMS-2728. Race/ethnicity was categorized as white, black, Hispanic, or other. Primary insurance at start of ESRD was categorized as private, Medicaid, none, or other (including Medicare, which is rare in SLE patients).

\section{Statistical analysis}

Patient characteristics were summarized overall and by attributed cause and vascular access at dialysis start. For analysis of placement of permanent access within the first year of dialysis, 40,911 patients with a catheter only at start of dialysis (Fig. 1) were included; for analysis of loss of patency of permanent access within the first year of dialysis, 13,073 patients with an AVF or AVG that was used on first dialysis (Fig. 1) were included.. The outcomes were time from dialysis start to first placement of AVF or AVG and time from start to first revision of the starting access or placement of a new access (Additional file 1: Table S1), with censoring for transplant, switch to peritoneal dialysis, death, or end of follow-up (1 year after dialysis start). Separate analyses for AVF and AVG placement were censored for placement of the other type of permanent access. Additional sensitivity analyses $(i)$ excluded children (age $<18$ years), to account for greater likelihood of pediatric ESRD onset in SLE- vs. non-SLE-ESRD; (ii) included only those with Medicare at ESRD start, to account for differential capture of events in the first 90 days of dialysis; (iii) included a comparison group of other glomerulonephritis patients [13], to examine whether SLE patients less likely than these potentially more comparable patients to have a permanent access placed; (iv) used 2:1 matching on age, sex, and race rather than statistical adjustment; and $(v)$ included all events prior to day 90 on day 91 and (vi) excluded all events prior to day 90 on the estimates obtained in primary analyses, also to account for potential differential capture of events prior to day 91 , when Medicare ESRD coverage for those patients $<65$ and not disabled begins. This scenario may be more likely in SLE, given patients' younger age at ESRD start. For both placement and loss of patency analyses, we generated Kaplan-Meier curves by SLE-ESRD vs. other ESRD and estimated hazard ratios (HRs) and CIs using crude and multivariable Cox proportional hazards models, with adjustment for age, sex, race/ethnicity, BMI, comorbid conditions, and smoking and using complete case analysis. Outcomes were not examined among the 13,494 remaining patients (Fig. 1) who had an access in place that was not used on first dialysis because the date of first use of the access cannot be determined from claims data. Stata v. 14 (StataCorp, College Station, TX) was used for all analyses and we adhered to STROBE guidelines for reporting.

\section{Results}

Characteristics of the study population by SLE status and vascular access at dialysis start

Patients with ESRD attributed to SLE were younger (mean age, 40.3 vs. 64.3 years), more likely to be female (79.3\% vs. $43.1 \%$ ) and black ( $54.9 \%$ vs. $29.8 \%$ ), and more likely to have Medicaid coverage at dialysis start (39.0 \% vs. $26.9 \%$ ), compared to patients with other attributed causes of ESRD $(P<0.001$ for all; Table 1). SLE-ESRD patients were less likely than other ESRD patients to have comorbid conditions and to smoke (Table 1). While low overall, transplantation $(2.5 \%$ vs. $1.3 \%, P=0.01)$ and renal recovery $(2.5 \%$ vs. $1.6 \%$; not statistically significant) within a year of dialysis start were both more likely in SLE-ESRD than other ESRD patients (Table 1). 
Table 1 Characteristics of 2010 incident U.S. hemodialysis patients, by vascular access at dialysis start and systemic lupus erythematosus status

\begin{tabular}{|c|c|c|c|c|c|c|c|c|c|}
\hline \multirow[t]{4}{*}{ Characteristic } & \multirow[t]{4}{*}{ Overall } & \multicolumn{8}{|c|}{ Vascular Access on First Dialysis: } \\
\hline & & \multicolumn{2}{|l|}{ Any } & \multicolumn{2}{|l|}{ Catheter only } & \multicolumn{2}{|l|}{ AVF/AVG in place } & \multicolumn{2}{|l|}{ AVF/AVG used } \\
\hline & & \multicolumn{8}{|c|}{ Attributed Cause of ESRD: } \\
\hline & & $\overline{S L E}$ & Other & SLE & Other & SLE & Other & SLE & Other \\
\hline $\mathrm{N}(\%)$ & $67,748(100 \%)$ & $517(0.8 \%)$ & $66,961(99.2 \%)$ & $384(0.9 \%)$ & $40,527(99.1 \%)$ & $85(0.6 \%)$ & $13,409(99.4 \%)$ & $48(0.4 \%)$ & $13,025(99.6 \%)$ \\
\hline \multicolumn{10}{|l|}{ Demographic } \\
\hline Mean (SD) age, years & $64.1(14.9)$ & $40.3(15.3)^{a}$ & $64.3(14.8)^{\mathrm{a}}$ & $39.0(15.0)^{a}$ & $64.1(15.3)^{\mathrm{a}}$ & $41.7(15.8)^{\mathrm{a}}$ & $63.8(14.3)^{\mathrm{a}}$ & $48.6(14.2)^{a}$ & $65.4(13.6)^{\mathrm{a}}$ \\
\hline Female, n (\%) & $29,276(43.4 \%)$ & $410(79.3 \%)^{a}$ & $28,866(43.1 \%)^{a}$ & $315(82.0 \%)^{a}$ & $17,951(44.3 \%)^{\mathrm{a}}$ & $64(75.3 \%)^{a}$ & $5,675(42.3 \%)^{a}$ & $31(64.6 \%)^{b}$ & $7,785(59.8 \%)^{b}$ \\
\hline \multicolumn{10}{|l|}{ Race/ethnicity, \% } \\
\hline Non-Hispanic white & $34,731(51.5 \%)$ & $129(25.0 \%)^{\mathrm{a}}$ & $34,602(51.7 \%)^{a}$ & $89(23.2 \%)^{a}$ & $20,813(51.4 \%)^{a}$ & $22(25.9 \%)^{a}$ & $6,644(49.6 \%)^{\mathrm{a}}$ & $18(37.5 \%)$ & $7,145(54.9 \%)$ \\
\hline Black & $20,221(30.0 \%)$ & $284(54.9 \%)^{\mathrm{a}}$ & $19,937(29.8 \%)^{a}$ & $221(57.6 \%)^{a}$ & $11,984(29.6 \%)^{a}$ & $42(49.4 \%)^{a}$ & $4,108(30.6 \%)^{a}$ & $21(43.8 \%)$ & $3,845(29.5 \%)$ \\
\hline Hispanic white & $8,722(12.9 \%)$ & $72(13.9 \%)^{a}$ & $8,650(12.9 \%)^{a}$ & $56(14.6 \%)^{a}$ & $5,526(13.6 \%)^{a}$ & $10(11.8 \%)^{a}$ & $1,829(13.6 \%)^{a}$ & $-^{\mathrm{c}}$ & 1,295 (9.9\%) \\
\hline Other & $3,804(5.6 \%)$ & $32(6.2 \%)^{a}$ & $3,772(5.6 \%)^{\mathrm{a}}$ & $18(4.7 \%)^{a}$ & $2,204(5.4 \%)^{a}$ & $11(12.9 \%)^{\mathrm{a}}$ & $828(6.2 \%)^{a}$ & $-^{c}$ & $740(5.7 \%)$ \\
\hline \multicolumn{10}{|l|}{ Insurance at dialysis start, \% } \\
\hline Private & $16,546(24.5 \%)$ & $137(26.5 \%)^{a}$ & $16,409(24.5 \%)^{a}$ & $93(24.2 \%)^{a}$ & $9,441(23.3 \%)^{a}$ & $25(29.4 \%)^{b}$ & $3,387(25.3 \%)^{b}$ & $19(39.6 \%)$ & 3,264 (25.1\%) \\
\hline Medicare/other & $27,159(40.3 \%)$ & $104(20.1 \%)^{a}$ & $27,055(40.4 \%)^{a}$ & $67(17.5 \%)^{\mathrm{a}}$ & $16,037(39.6 \%)^{\mathrm{a}}$ & $21(24.7 \%)^{b}$ & $5,315(39.6 \%)^{b}$ & 16 (33.3 \%) & $3,581(27.5 \%)$ \\
\hline Medicaid & $18,183(27.0 \%)$ & $201(38.9 \%)^{a}$ & $17.982(26.9 \%)^{a}$ & $160(41.7 \%)^{a}$ & $10,897(26.9 \%)^{a}$ & $32(37.7 \%)^{b}$ & $3,821(28.5 \%)^{\mathrm{b}}$ & $-^{c}$ & $5,703(43.8 \%)$ \\
\hline None & $5,590(8.3 \%)$ & $75(14.5 \%)^{a}$ & $5,515(8.2 \%)^{\mathrm{a}}$ & $64(16.7 \%)^{a}$ & $4,152(10.3 \%)^{a}$ & $-^{c}$ & $886(6.6 \%)^{b}$ & $-^{c}$ & 477 (3.7\%) \\
\hline \multicolumn{10}{|l|}{ Clinical } \\
\hline Pre-ESRD nephrology care, \% & $38,937(65.7 \%)$ & $307(67.0 \%)$ & $38,630(65.7 \%)$ & $200(60.2 \%)^{b}$ & $18,042(52.4 \%)^{\mathrm{b}}$ & $64(81.0 \%)$ & $9,034(74.4 \%)$ & $43(91.5 \%)$ & $11,554(94.3 \%)$ \\
\hline Mean (SD) BMl, kg/m² & $29.5(7.9)$ & $27.3(7.5)^{\mathrm{a}}$ & $29.5(7.9)^{\mathrm{a}}$ & $27.2(7.5)^{a}$ & $29.3(8.0)^{\mathrm{a}}$ & $27.7(6.9)^{\mathrm{a}}$ & $29.9(7.9)^{a}$ & $27.3(8.3)^{b}$ & $29.8(7.7)^{\mathrm{b}}$ \\
\hline Congestive heart failure, $\%$ & $22,893(33.9 \%)$ & $85(16.4 \%)^{a}$ & $22,808(34.1 \%)^{a}$ & $59(15.4 \%)^{a}$ & $14,292(35.3 \%)^{\mathrm{a}}$ & $18(21.2 \%)^{b}$ & $4,819(35.9 \%)^{b}$ & $-^{c}$ & $3,697(28.4 \%)$ \\
\hline Diabetes, $\%$ & $38,918(57.7 \%)$ & $62(12.0 \%)^{\mathrm{a}}$ & $38,856(58.0 \%)^{a}$ & $44(11.5 \%)^{\mathrm{a}}$ & $22,940(56.6 \%)^{a}$ & $12(14.1 \%)^{a}$ & $8,401(62.7 \%)^{a}$ & $-^{c}$ & $7,515(57.7 \%)^{a}$ \\
\hline Peripheral vascular disease, $\%$ & 9,569 (14.2\%) & $21(4.1 \%)^{a}$ & $9,548(14.3 \%)^{a}$ & $16(4.2 \%)^{a}$ & $5,494(13.6 \%)^{\mathrm{a}}$ & $-^{c}$ & $2,267(16.9 \%)^{b}$ & $-^{c}$ & $1,787(13.7 \%)^{b}$ \\
\hline Smoking, \% & $4,369(6.5 \%)$ & $26(5.0 \%)$ & $4,343(6.5 \%)$ & $13(3.4 \%)^{b}$ & $2,645(6.5 \%)^{b}$ & $10(11.8 \%)$ & $889(6.6 \%)$ & $-^{c}$ & $810(6.2 \%)$ \\
\hline Transplanted within 1 year, \% & $853(1.3 \%)$ & $13(2.5 \%)^{b}$ & $840(1.3 \%)^{b}$ & $-^{c}$ & $429(1.1 \%)^{b}$ & $-^{c}$ & $160(1.2 \%)$ & $-^{c}$ & $252(1.9 \%)$ \\
\hline Recovered renal function within 1 year, \% & $1048(1.6 \%)$ & $13(2.5 \%)$ & $1035(1.6 \%)$ & $11(2.9 \%)$ & 909 (2.2 \%) & $-^{c}$ & $85(0.6 \%)$ & $-^{c}$ & 41 (0.3\%) \\
\hline
\end{tabular}

AVF arteriovenous fistula, $A V G$ arteriovenous graft, ESRD end-stage renal disease, SLE systemic lupus eythematosus

${ }^{\mathrm{a}} P<0.001$ and ${ }^{\mathrm{b}} P<0.05$ for SLE vs. other ESRD, by $t$ or chi-square/Fisher's exact test, as appropriate

${ }^{c}$ Not reportable due to cell size $<10$ 
Most SLE-ESRD patients (74.3 \%) started dialysis with only a catheter, while $9.3 \%$ started dialysis using an AVF or AVG; in contrast, $60.5 \%$ and $19.5 \%$ of other ESRD patients started dialysis with a catheter only or using an AVF/AVG, respectively $(P<0.001)$. Within subgroups defined by starting vascular access, patterns by SLE status of patient characteristics were generally similar to the patterns in the overall dialysis patients (Table 1). For those using an AVF/AVG on first dialysis, differences by SLE status by female sex and black race were slightly attenuated, relative to the overall population (Table 1). SLE patients starting dialysis with an AVF/AVG were older (mean age, 48.6 years) and more likely to have private insurance than other SLE-ESRD patients (Table 1).

\section{Association of SLE status with vascular access outcomes Vascular access placement within 1 year}

Among hemodialysis patients without a permanent access at dialysis start, $46.1 \%$ of SLE-ESRD patients and $54.5 \%$ of other ESRD patients had a permanent access placed within 1 year of dialysis start (Table 2). Crude rates of AVF/AVG placement over the first year of dialysis were $26 \%$ lower among patients with SLE-ESRD who did not have a permanent access in place at dialysis start, relative to their counterparts with other ESRD (Fig. 2a; Table 2). However, adjustment for demographics and clinical characteristics rendered this association null (Table 2), and this attenuation was largely driven by adjustment for differences in ages between SLE-ESRD and other ESRD patients [age-adjusted HR: 0.96 (95\% CI, 0.83-1.12)]. Similar patterns were seen for AVF and AVG placement individually, although crude results were not statistically significant for AVG placement alone (Table 2). Sensitivity analyses showed results similar to those obtained in the primary analysis (Table 3 ).

\section{Loss of vascular access patency in first year of dialysis}

SLE-ESRD patients using a permanent access (AVF/ AVG) on first dialysis were less likely than their non-SLE counterparts to experience a loss of patency of that access within the first year $(43.8 \%$ vs. $55.0 \%, P=0.12$; Table 4). SLE-ESRD patients had $30 \%$ lower crude rates of access revision than other ESRD patients within the first year of dialysis, but the association was not statistically significant (Fig. 2b; Table 4). Adjustment for potential confounders attenuated the association. Results among those starting with an AVF were similar to the overall results, whereas results among those starting with an AVG showed a greater magnitude of effect ( $46 \%$ lower rates of access revisions among SLE-ESRD vs. other ESRD patients) but without statistical significance, likely due to low numbers of individuals and events in the SLE-ESRD subgroup (Table 4). Results from sensitivity analyses excluding children were similar to results from the primary analysis (Table 5).

\section{Discussion}

We found that, among U.S. hemodialysis patients starting treatment in 2010 without a permanent access in place, those with SLE-ESRD were equally as likely as other ESRD patients to have an AVF or AVG placed in the first year of dialysis after adjustment for potential confounders, particularly age. These results, which were robust to sensitivity analyses, suggest that the differential prevalence of permanent access in place at the start of

Table 2 Association of placement of permanent vascular access with attribution of end-stage renal disease to systemic lupus erythematosus vs. other causes, among U.S. incident hemodialysis patients who started dialysis in 2010 with only a catheter in place

\begin{tabular}{|c|c|c|c|c|}
\hline \multirow[t]{2}{*}{ Model } & \multirow{2}{*}{$\begin{array}{l}\text { No. (\%) with permanent } \\
\text { access placed within } 1 \text { year }\end{array}$} & \multicolumn{3}{|c|}{ Hazard ratio $(95 \% \mathrm{Cl})$ for placement of permanent vascular access, SLE-attributed vs. other ESRD } \\
\hline & & Unadjusted & Adjusted for demographics & Adjusted for demographics + clinical \\
\hline \multicolumn{5}{|l|}{ All events } \\
\hline SLE-attributed ESRD & $177(46.1 \%)$ & $0.74(0.64-0.86)$ & $0.94(0.81-1.09)$ & $1.00(0.86-1.17)$ \\
\hline Other ESRD & $22,076(54.5 \%)$ & 1.00 (ref.) & 1.00 (ref.) & 1.00 (ref.) \\
\hline$P$ & 0.001 & $<0.001$ & 0.42 & $>0.9$ \\
\hline \multicolumn{5}{|l|}{ All AVF placements ${ }^{\mathrm{a}}$} \\
\hline SLE-attributed ESRD & $137(35.7 \%)$ & $0.73(0.62-0.87)$ & $0.93(0.79-1.11)$ & $1.00(0.84-1.18)$ \\
\hline Other ESRD & 17,300 (42.7 \%) & 1.00 (ref.) & 1.00 (ref.) & 1.00 (ref.) \\
\hline$P$ & 0.006 & $<0.001$ & 0.43 & $>0.9$ \\
\hline \multicolumn{5}{|l|}{ All AVG placements ${ }^{a}$} \\
\hline SLE-attributed ESRD & 40 (10.4\%) & $0.77(0.56-1.05)$ & $1.00(0.73-1.38)$ & $1.06(0.77-1.47)$ \\
\hline Other ESRD & 4,776 (11.8\%) & 1.00 (ref.) & 1.00 (ref.) & 1.00 (ref.) \\
\hline$P$ & 0.41 & 0.10 & $>0.9$ & 0.71 \\
\hline
\end{tabular}

AVF arteriovenous fistula, AVG arteriovenous graft, ESRD end-stage renal disease, SLE systemic lupus eythematosus. Demographics: age (continuous), sex, race; clinical: body mass index (continuous), smoking, congestive heart failure, diabetes, and peripheral vascular disease

${ }^{\text {a } T i m e-t o-e v e n t ~ a n a l y s e s ~ c e n s o r e d ~ f o r ~ p l a c e m e n t ~ o f ~ o t h e r ~ t y p e ~ o f ~ a c c e s s ~}$ 


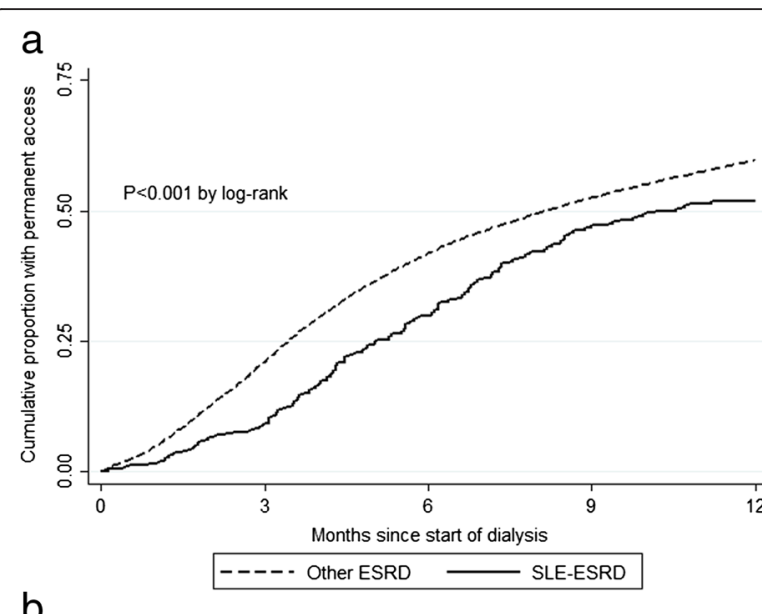

b

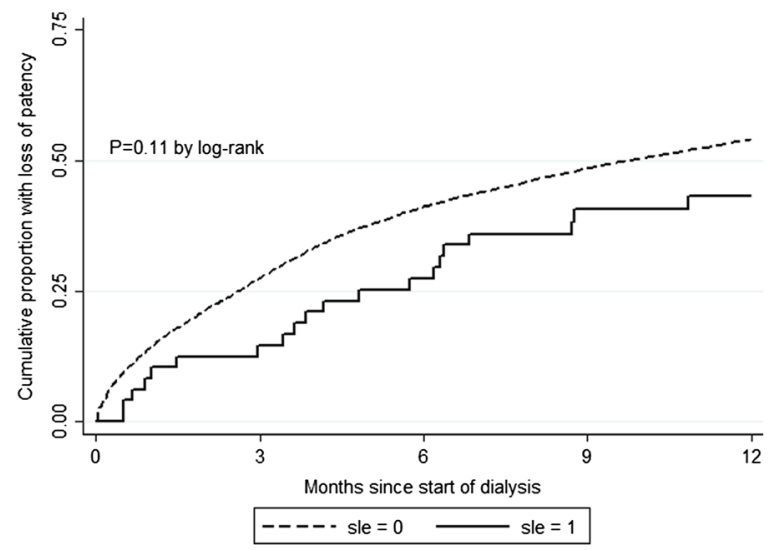

Fig. 2 Time to access placement $\mathbf{a}$ and loss of access patency $\mathbf{b}$ in the first year of dialysis, among U.S. incident hemodialysis patients with systemic lupus erythematosus-attributed vs. other end-stage renal disease in 2010. a, among patients who started with only a catheter; $\mathbf{b}$, among patients who used an arteriovenous fistula or graft on first dialysis. ESRD, end-stage renal disease; SLE, systemic lupus erythematosus

hemodialysis seen in prior studies of both adults (24\% vs. $36 \%$ for SLE vs. other ESRD patients) [11-13] and children (23\% vs. $43 \%$ ) $[15,16]$ is not the result of SLEESRD patients being more likely than other ESRD patients to have their permanent access placed after, rather than before, hemodialysis start. Further, it suggests that the substantial gap in permanent access placement by SLE status at ESRD start does not narrow, even after a year on treatment, which may subject SLE-ESRD patients receiving hemodialysis to greater risk of morbidity and mortality [1-8] than their non-SLE counterparts.

One reason providers and patients might delay placement of permanent access in a patient approaching ESRD treatment that the patient is expected to receive a kidney transplant shortly after hemodialysis treatment begins. This scenario may be more common in SLEESRD patients, who tend to be younger and, potentially, better transplant candidates, as suggested by their relatively greater rates of placement on the deceased donor waitlist and transplantation [12, 17]. Here, we found that, while early transplantation was nearly twice as common among the SLE-ESRD vs. other ESRD patients with only a catheter for access at the start of hemodialysis, the absolute likelihood of transplantation within the first year of hemodialysis among these SLE-ESRD patients was only $2.5 \%$, suggesting that expected early transplantation is not a justification for lack of permanent access placement at or before hemodialysis start. Similarly, providers may be more likely to anticipate renal recovery in SLE-ESRD [18] and avoid vascular surgery referral, but we found that, while SLE patients who started dialysis with a catheter only were more likely to recover renal function within a year than their non-SLE counterparts, fewer than $3 \%$ of these SLE patients recovered renal function. Thus, anticipated recovery is also not likely to play a large role in the continuing gap in permanent vascular access placement by SLE status among hemodialysis patients after the first year of hemodialysis treatment. The predominance of female sex among SLEESRD patients might be another reason for the continued disparity in vascular access placement by SLE status over the first year of hemodialysis, due to potentially greater difficulty placing AVFs [19] and greater prevalence of body image issues associated with permanent vascular access [20] among females. However, adjustment for sex did not change our results. Differential provider referrals for vascular access surgery by SLE status could be also affected by greater likelihood of intravenous treatment history and/or hypercoagulability in SLE patients, particularly those who may have anti-phospholipid syndrome [21]; data were not available to examine these potential factors.

We also found that, among hemodialysis patients who started treatment with a permanent vascular access, loss of patency of the vascular access was actually less common among SLE-ESRD than other ESRD patients. However, this association was not statistically significant, even before adjustment. These results suggest that any provider perceptions about increased risk of vascular access problems for SLE-ESRD patients vs. other ESRD patients on hemodialysis due to clinical features of SLE [21], which could lead to fewer referrals to vascular access surgery, could be overestimated or even unfounded. However, these results should be interpreted with caution due to the potential for confounding by indication. In other words, it is possible that SLE-ESRD patients who started hemodialysis with a permanent vascular access were referred for vascular access surgery because they were at lesser risk for loss of vascular access patency than other SLE-ESRD patients, due to clinical features of their SLE not captured in these administrative data. Thus, we can only conclude that, among those ESRD patients who are observed to have a permanent access placed prior to the start of hemodialysis, SLE status is likely not associated with greater risk of loss of patency. 
Table 3 Association of placement of permanent vascular access with attribution of end-stage renal disease to systemic lupus erythematosus vs. other causes, among U.S. incident hemodialysis patients who started dialysis in 2010 with only a catheter in place: sensitivity analyses

\begin{tabular}{|c|c|c|c|c|}
\hline \multirow[t]{2}{*}{ Model } & \multirow[t]{2}{*}{$\begin{array}{l}\text { No. (\%) with permanent } \\
\text { access placed within } 1 \text { year }\end{array}$} & \multicolumn{3}{|c|}{$\begin{array}{l}\text { Hazard ratio }(95 \% \mathrm{Cl}) \text { for placement of permanent vascular access, } \\
\text { SLE-attributed vs. Other ESRD }\end{array}$} \\
\hline & & Unadjusted & Adjusted for demographics & Adjusted for demographics + clinical \\
\hline \multicolumn{5}{|l|}{ Primary analysis } \\
\hline SLE-attributed ESRD & $177(46.1 \%)$ & $0.74(0.64-0.86)$ & $0.94(0.81-1.09)$ & $1.00(0.86-1.17)$ \\
\hline Other ESRD & $22,076(54.5 \%)$ & 1.00 (ref.) & 1.00 (ref.) & 1.00 (ref.) \\
\hline P & 0.001 & $<0.001$ & 0.42 & $>0.9$ \\
\hline \multicolumn{5}{|c|}{ Excluding patients $<18$ years old ${ }^{a}$} \\
\hline SLE-attributed ESRD & $171(46.2 \%)$ & $0.74(0.64-0.86)$ & $0.92(0.79-1.08)$ & $0.98(0.84-1.15)$ \\
\hline Other ESRD & $22,053(54.6 \%)$ & 1.00 (ref.) & 1.00 (ref.) & 1.00 (ref.) \\
\hline$P$ & 0.001 & $<0.001$ & 0.31 & 0.82 \\
\hline \multicolumn{5}{|l|}{$\begin{array}{l}\text { Only among patients with } \\
\text { Medicare at ESRD start }\end{array}$} \\
\hline SLE-attributed ESRD & $67(58.3 \%)$ & $0.77(0.60-0.99)$ & $0.83(0.65-1.07)$ & $0.83(0.65-1.07)$ \\
\hline Other ESRD & $15,635(64.0 \%)$ & 1.00 (ref.) & 1.00 (ref.) & 1.00 (ref.) \\
\hline$P$ & 0.20 & 0.04 & 0.15 & 0.15 \\
\hline \multicolumn{5}{|l|}{$\begin{array}{l}\text { SLE and other GN compared } \\
\text { to other ESRD }\end{array}$} \\
\hline SLE-attributed ESRD & $177(46.1 \%)$ & $0.73(0.63-0.85)$ & $0.93(0.80-1.08)$ & $0.97(0.83-1.14)$ \\
\hline Other GN-attributed ESRD & $1,219(48.6 \%)$ & $0.83(0.78-0.88)$ & $0.91(0.86-0.96)$ & $0.94(0.89-1.00)$ \\
\hline Other ESRD & $20,857(54.9 \%)$ & 1.00 (ref.) & 1.00 (ref.) & 1.00 (ref.) \\
\hline$P$ & $<0.001$ & $<0.001 /<0.001$ & $0.35 /<0.001$ & $0.74 / 0.06$ \\
\hline \multicolumn{5}{|l|}{ Matched analysis $^{d}$} \\
\hline SLE-attributed ESRD & $177(46.1 \%)$ & $0.85(0.71-1.02)$ & $0.88(0.73-1.06)$ & $0.93(0.77-1.13)$ \\
\hline Other ESRD & $350(51.7 \%)$ & 1.00 (ref.) & 1.00 (ref.) & 1.00 (ref.) \\
\hline P & 0.08 & 0.09 & 0.17 & 0.48 \\
\hline \multicolumn{5}{|c|}{$\begin{array}{l}\text { Events prior to } 90 \text { days included } \\
\text { on day } 91\end{array}$} \\
\hline SLE-attributed ESRD & 177 (46.1 \%) & $0.76(0.65-0.88)$ & $0.94(0.81-1.10)$ & $1.00(0.86-1.17)$ \\
\hline Other ESRD & $22,076(54.5 \%)$ & 1.00 (ref.) & 1.00 (ref.) & 1.00 (ref.) \\
\hline$P$ & 0.001 & $<0.001$ & 0.46 & $>0.9$ \\
\hline \multicolumn{5}{|c|}{ Events prior to 90 days excluded ${ }^{e}$} \\
\hline SLE-attributed ESRD & $142(40.7 \%)$ & $0.93(0.79-1.10)$ & $1.05(0.89-1.25)$ & $1.07(0.91-1.27)$ \\
\hline Other ESRD & $13,653(42.6 \%)$ & 1.00 (ref.) & 1.00 (ref.) & 1.00 (ref.) \\
\hline$P$ & 0.48 & 0.41 & 0.56 & 0.42 \\
\hline
\end{tabular}

AVF arteriovenous fistula, AVG arteriovenous graft, ESRD end-stage renal disease, GN glomerulonephritis, SLE systemic lupus eythematosus. Demographics: age (continuous), sex, race; clinical: body mass index (continuous), smoking, congestive heart failure, diabetes, and peripheral vascular disease

aExcluding $n=162$ patients (14 SLE, 148 other) who were $<18$ years old

${ }^{\mathrm{b}}$ Excluding $n=16,374$ patients (269 SLE, 16,105 other) without Medicare at ESRD start

'Other GN included 2,511 patients with CMS-2728 ICD-9 codes for attributed cause of glomerulonephritis (582.9, 582.1, 583.1, 583.21, 583.22, 583.81, 583.82, 583.4, 580.0 , and 582.0) or secondary glomerulonephritis/vasculitis (excluding SLE-ESRD; 287.0, 710.1, 283.11, 446.0, 446.4, 583.92, 446.20, 446.21, and 583.91)

${ }^{\mathrm{d}}$ Among $n=1061$ patients, including 384 SLE patients and 677 matched non-SLE patients, using 2:1 matching on age group ( $<30,30-49$, and $>50$ years), sex (female and male), and race (black and not black). Models stratified on matching variables to account for matching

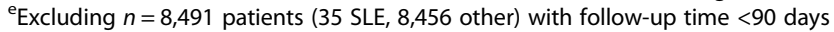

We found that AVFs were placed more often than AVGs in both SLE-ESRD and other ESRD patients in the first year of dialysis, which is in line with national clinical practice guidelines [9] and quality improvement programs such as the Fistula First, Catheter Last initiative [10]. However, the null association of permanent vascular access placement with SLE status did not differ by type of permanent access placed. We also found that 
Table 4 Association of loss of vascular access patency ${ }^{a}$ with attribution of ESRD to SLE vs. other causes, among 2010 U.S. incident hemodialysis patients who started dialysis with a permanent vascular access

\begin{tabular}{|c|c|c|c|c|}
\hline \multirow[t]{2}{*}{ Model } & \multirow{2}{*}{$\begin{array}{l}\text { No. (\%) with access } \\
\text { revision within } 1 \text { year }\end{array}$} & \multicolumn{3}{|c|}{ Hazard ratio $(95 \% \mathrm{Cl})$ for loss of patency, SLE-attributed vs. other ESRD } \\
\hline & & Unadjusted & Adjusted for demographics & Adjusted for demographics + clinical \\
\hline \multicolumn{5}{|l|}{ All patients } \\
\hline SLE-attributed ESRD & $21(43.8 \%)$ & $0.70(0.45-1.09)$ & $0.81(0.52-1.26)$ & $0.88(0.57-1.37)$ \\
\hline Other ESRD & $7,169(55.0 \%)$ & 1.00 (ref.) & 1.00 (ref.) & 1.00 (ref.) \\
\hline$P$ & 0.12 & 0.11 & 0.35 & 0.58 \\
\hline \multicolumn{5}{|c|}{ Among patients with $A V F^{b}$} \\
\hline SLE-attributed ESRD & 19 (44.2\%) & $0.74(0.46-1.17)$ & $0.86(0.54-1.37)$ & $0.94(0.59-1.50)$ \\
\hline Other ESRD & $5,735(52.9 \%)$ & 1.00 (ref.) & 1.00 (ref.) & 1.00 (ref.) \\
\hline$P$ & 0.26 & 0.20 & 0.53 & 0.80 \\
\hline \multicolumn{5}{|c|}{ Among patients with $A V G^{b}$} \\
\hline SLE-attributed ESRD & $-^{c}$ & $0.54(0.13-2.15)$ & $0.57(0.14-2.30)$ & $0.61(0.15-2.47)$ \\
\hline Other ESRD & 1,434 (65.8 \%) & 1.00 (ref.) & 1.00 (ref.) & 1.00 (ref.) \\
\hline$P$ & 0.35 & 0.38 & 0.43 & 0.49 \\
\hline
\end{tabular}

$A V F$ arteriovenous fistula, AVG arteriovenous graft, ESRD end-stage renal disease, SLE systemic lupus eythematosus. Demographics: age (continuous), sex, race; clinical: body mass index (continuous), smoking, congestive heart failure, diabetes, and peripheral vascular disease

a Defined as a revision or placement of a new AVF, AVG, or catheter (see Table 1)

${ }^{\mathrm{b}} \mathrm{N}=10,890$ and 2,183 for AVF and AVG, respectively

${ }^{\mathrm{C}}$ Not reportable due to cell size $<10$

loss of vascular access patency was more common among patients starting hemodialysis with AVGs vs. AVFs, which is consistent with prior evidence that access outcomes are poorer in AVGs than AVFs [1,22, 23]. Results were suggestive that SLE-ESRD patients with AVGs were less likely to experience loss of patency than other ESRD patients with AVGs, whereas there was no association of SLE status with revisions among patients with AVFs. However, differences by SLE status were not statistically significant, likely due to lack of power in subgroup analyses.

This study has several important strengths, including the capture of all U.S. patients treated for ESRD, limited loss to follow-up due to universal coverage of ESRD services by CMS, completion of the CMS-2728 for all treated patients, and availability of claims data for all inpatient and outpatient services after start of dialysis. However, there are limitations, in addition to those noted above, which deserve mention. There is the potential for selection bias in the exclusion of a large number of patients without Medicare Part B coverage, and SLE-ESRD patients were more likely than other ESRD patients (33.5 \% vs. $25.4 \%$ ) to be excluded due to lack of Part B coverage. This exclusion, while necessary to include outpatient events, also limits generalizability to the entire U.S. ESRD population. There is also the potential for misclassification of the outcomes using claims data. We cannot address the question of whether SLE-ESRD and other ESRD patients who start hemodialysis with a permanent access in place at dialysis start have the same rates of access maturation and eventual use, because administrative data do not capture which access was used during each dialysis session. As with all observational studies, residual confounding by unmeasured factors such as differences in training and experience of regional vascular access surgeons [24] is possible. There is also the possibility of overadjustment, given that some variables may be mediators as well as confounders and that our power was limited due to small sample sizes in some subgroups, such as SLE patients with AVGs. However, we found that, after adjustment for age, further adjustment did not change results substantially. Sensitivity of the attribution of ESRD cause to SLE on the CMS-2728 has been suggested to be low [25], although our more recent study suggested much higher sensitivity (79 \%) for the capture of U.S. patients with a validated diagnosis of SLE who have progressed to ESRD [26]; however, many SLE patients may remain in the comparison group. Misclassification of access revision could have occurred by including placement of a new access in the definition, since accesses may be placed for other reasons, including aneurysm formation and steal syndrome. Data are from 2010 and may not reflect current clinical practice regarding vascular access placement and revisions generally, or specifically in SLE patients. Finally, while we performed multiple sensitivity analyses, we cannot fully address the possibility of missed events in the first 90 days of dialysis, which may differentially occur in SLE-ESRD patients.

\section{Conclusion}

We found that SLE-ESRD patients were not more likely to have a permanent access placed in the first year of dialysis, despite a substantial gap in access placement at the start of hemodialysis [12] and despite an observed lack of association of SLE status with subsequent loss of 
Table 5 Association of loss of vascular access patency ${ }^{a}$ with attribution of ESRD to SLE vs. Other causes, among 2010 U.S. incident hemodialysis patients who started dialysis with a permanent vascular access: sensitivity analyses

\begin{tabular}{|c|c|c|c|c|}
\hline \multirow[t]{2}{*}{ Model } & \multirow{2}{*}{$\begin{array}{l}\text { No. (\%) with access } \\
\text { revision within } 1 \text { year }\end{array}$} & \multicolumn{3}{|c|}{ Hazard ratio $(95 \%$ Cl) for loss of patency, SLE-attributed vs. other ESRD } \\
\hline & & Unadjusted & Adjusted for demographics & Adjusted for demographics + clinical \\
\hline \multicolumn{5}{|l|}{ Primary analysis } \\
\hline SLE-attributed ESRD & $21(43.8 \%)$ & $0.70(0.45-1.09)$ & $0.81(0.52-1.26)$ & $0.88(0.57-1.37)$ \\
\hline Other ESRD & $7,169(55.0 \%)$ & 1.00 (ref.) & 1.00 (ref.) & 1.00 (ref.) \\
\hline$P$ & 0.12 & 0.11 & 0.35 & 0.58 \\
\hline \multicolumn{5}{|c|}{ Excluding patients $<18$ years old ${ }^{\mathrm{b}}$} \\
\hline SLE-attributed ESRD & $21(43.8 \%)$ & $0.70(0.45-1.08)$ & $0.81(0.52-1.26)$ & $0.88(0.57-1.37)$ \\
\hline Other ESRD & $7,165(55.1 \%)$ & 1.00 (ref.) & 1.00 (ref.) & 1.00 (ref.) \\
\hline$P$ & 0.12 & 0.11 & 0.35 & 0.57 \\
\hline \multicolumn{5}{|l|}{$\begin{array}{l}\text { Only among patients with } \\
\text { Medicare at ESRD start }{ }^{c}\end{array}$} \\
\hline SLE-attributed ESRD & $-^{d}$ & $0.91(0.55-1.52)$ & $0.86(0.52-1.42)$ & $0.92(0.55-1.53)$ \\
\hline Other ESRD & 1125 (13.0\%) & 1.00 (ref.) & 1.00 (ref.) & 1.00 (ref.) \\
\hline$P$ & 0.76 & 0.73 & 0.55 & 0.75 \\
\hline \multicolumn{5}{|l|}{$\begin{array}{l}\text { SLE and other GN compared } \\
\text { to other ESRD }\end{array}$} \\
\hline SLE-attributed ESRD & $-^{d}$ & $0.69(0.44-1.07)$ & $0.80(0.52-1.24)$ & $0.87(0.56-1.35)$ \\
\hline Other GN-attributed ESRD & $60(6.9 \%)$ & $0.79(0.71-0.88)$ & $0.87(0.78-0.96)$ & $0.91(0.82-1.02)$ \\
\hline Other ESRD & $1433(11.8 \%)$ & 1.00 (ref.) & 1.00 (ref.) & 1.00 (ref.) \\
\hline$P$ & $<0.001$ & $0.10 /<0.001$ & $0.32 / 0.008$ & $0.54 / 0.09$ \\
\hline \multicolumn{5}{|l|}{ Matched analysis $^{f}$} \\
\hline SLE-attributed ESRD & $21(43.8 \%)$ & $0.71(0.42-1.19)$ & $0.70(0.42-1.15)$ & $0.81(0.47-1.40)$ \\
\hline Other ESRD & $89(58.2 \%)$ & 1.00 (ref.) & 1.00 (ref.) & 1.00 (ref.) \\
\hline$P$ & 0.08 & 0.19 & 0.16 & 0.46 \\
\hline \multicolumn{5}{|c|}{$\begin{array}{l}\text { Events prior to } 90 \text { days included } \\
\text { on day } 91\end{array}$} \\
\hline SLE-attributed ESRD & $21(43.8 \%)$ & $0.73(0.47-1.13)$ & $0.81(0.52-1.27)$ & $0.88(0.57-1.37)$ \\
\hline Other ESRD & 7,169 (55.0\%) & 1.00 (ref.) & 1.00 (ref.) & 1.00 (ref.) \\
\hline$P$ & 0.12 & 0.16 & 0.36 & 0.57 \\
\hline \multicolumn{5}{|c|}{ Events prior to 90 days excluded ${ }^{9}$} \\
\hline SLE-attributed ESRD & $13(32.5 \%)$ & $0.85(0.49-1.46)$ & $0.76(0.44-1.31)$ & $0.84(0.48-1.45)$ \\
\hline Other ESRD & 3,377 (36.6 \%) & 1.00 (ref.) & 1.00 (ref.) & 1.00 (ref.) \\
\hline$P$ & 0.59 & 0.56 & 0.32 & 0.52 \\
\hline
\end{tabular}

AVF arteriovenous fistula, AVG arteriovenous graft, ESRD end-stage renal disease, GN glomerulonephritis, SLE systemic lupus eythematosus. Demographics: age (continuous), sex, race; clinical: body mass index (continuous), smoking, congestive heart failure, diabetes, and peripheral vascular disease

${ }^{a}$ Defined as a revision or placement of a new AVF, AVG, or catheter (see Table 1)

${ }^{\mathrm{b} E x c l u d i n g} n=14$ patients (4 SLE, 10 other) who were $<18$ years old

'Excluding $n=4,375$ patients (23 SLE, 4,352 other) without Medicare at ESRD start

${ }^{\mathrm{d}}$ Not reportable due to cell size $<10$

e Other GN included 864 patients with CMS-2728 ICD-9 codes for attributed cause of glomerulonephritis $(582.9,582.1,583.1,583.21,583.22,583.81,583.82,583.4$ 580.0, and 582.0) or secondary glomerulonephritis/vasculitis (excluding SLE-ESRD; 287.0, 710.1, 283.11, 446.0, 446.4, 583.92, 446.20, 446.21, and 583.91)

${ }_{\mathrm{f}}^{\mathrm{f}} \mathrm{m}$ mong $n=201$ patients, including 48 SLE patients and 153 matched non-SLE patients, using 2:1 matching on age group ( $<30,30-49$, and $>50$ years), sex (female and male), and race (black and not black). Models stratified on matching variable to account for matching

${ }^{9}$ Excluding $n=3,801$ patients (35 SLE, 8,456 other) with follow-up time $<90$ days

access patency. SLE-ESRD patients are seen by multiple providers, potentially increasing opportunities for shared decision-making and coordinated care; yet, this population does not have adequate hemodialysis vascular access. Future studies should focus on provider and patient perceptions of permanent vascular access for hemodialysis in SLE-ESRD and, more generally, on the roles of nephrologists, rheumatologists, and patients in ensuring high-quality care and optimal outcomes in hemodialysis. 


\section{Additional file}

Additional file 1: Table S1. Healthcare Common Procedure Coding System codes used to identify vascular access events within 1 year of dialysis start in U.S. patients initiating hemodialysis in 2010. (DOCX 15 kb)

\section{Abbreviations}

AVF, arteriovenous fistula; AVG, arteriovenous graft; BMI, body mass index CMS, Centers for Medicare \& Medicaid Services; ESRD, end-stage renal disease; HCPCS, healthcare common procedure coding system; SLE, systemic lupus erythematosus

\section{Acknowledgements}

Portions of this work were presented at the American Society of Nephrology Kidney Week 2015, held November 3-8, 2015, in San Diego, California. The data reported here have been supplied by the United States Renal Data System (USRDS). The interpretation and reporting of these data are the responsibility of the authors and in no way should be seen as an official policy or interpretation of the U.S. government.

\section{Funding}

LCP, REP, and SOP are supported in part by 1R01MD 010290 through the National Institute on Minority Health and Health Disparities. CD and SSL are supported in part by NIH R01AR065493 and CDC U01DP005119. The content is solely the responsibility of the authors and does not necessarily represent the official views of the $\mathrm{NIH}$.

\section{Availability of data and materials}

The datasets supporting the conclusions of this article are available via request from the United States Renal Data System (www.usrds.org).

\section{Authors' contributions}

LCP conceived the study, analyzed the data, and drafted the manuscript. SSL, SOP, and CD provided substantive clinical and scientific input on each version of the manuscript. REP acquired the data and provided substantive scientific input on each version of the manuscript. All authors read and approved the final manuscript.

\section{Competing interests}

The authors declare that they have no competing interests.

\section{Consent for publication}

Not applicable; informed consent was waived for this retrospective study of limited data.

\section{Ethics approval and consent to participate}

This study was approved by the Emory Institutional Review Board. Consent was waived for this this retrospective study of limited data.

\section{Author details}

'Division of Renal Medicine, Department of Medicine, Emory University, 101 Woodruff Circle, 5105 Woodruff Memorial Building, Atlanta, GA 30322, USA. ${ }^{2}$ Division of Rheumatology, Department of Medicine, Emory University, Atlanta, GA, USA. ${ }^{3}$ Division of Transplantation, Department of Surgery, Emory University, Atlanta, GA, USA. ${ }^{4}$ Emory Transplant Center, Emory Healthcare, Emory University, Atlanta, GA, USA

Received: 24 February 2016 Accepted: 8 June 2016 Published online: 07 July 2016

\section{References}

1. Pisoni RL, Young EW, Dykstra DM, Greenwood RN, Hecking E, Gillespie B, Wolfe RA, Goodkin DA, Held PJ. Vascular access use in Europe and the United States: results from the DOPPS. Kidney Int. 2002:61(1):305-16.

2. Feldman $\mathrm{HI}$, Kobrin S, Wasserstein A. Hemodialysis vascular access morbidity. J Am Soc Nephrol. 1996;7(4):523-35.

3. Dhingra RK, Young EW, Hulbert-Shearon TE, Leavey SF, Port FK. Type of vascular access and mortality in U.S. hemodialysis patients. Kidney Int. 2001; 60(4):1443-51.
4. Woods JD, Port FK. The impact of vascular access for haemodialysis on patient morbidity and mortality. Nephrol Dial Transplant. 1997:12(4):657-9.

5. Xue JL, Dahl D, Ebben JP, Collins AJ. The association of initial hemodialysis access type with mortality outcomes in elderly Medicare ESRD patients. Am J Kidney Dis. 2003;42(5):1013-9.

6. Polkinghorne KR, McDonald SP, Atkins RC, Kerr PG. Vascular access and all-cause mortality: a propensity score analysis. J Am Soc Nephrol. 2004;15(2):477-86.

7. Pastan S, Soucie JM, McClellan WM. Vascular access and increased risk of death among hemodialysis patients. Kidney Int. 2002;62(2):620-6.

8. Lee H, Manns B, Taub K, Ghali WA, Dean S, Johnson D, Donaldson C. Cost analysis of ongoing care of patients with end-stage renal disease: the impact of dialysis modality and dialysis access. Am J Kidney Dis. 2002;40(3):611-22.

9. Vascular Access 2006 Work Group. Clinical practice guidelines for vascular access. Am J Kidney Dis. 2006;48(Suppl 1):S176-247.

10. End Stage Renal Disease (ESRD) National Coordinating Center (NCC). Fistula First, Catheter Last. Available at: http://esrdncc.org/ffcl/.

11. Plantinga LC, Drenkard C, Patzer RE, Klein M, Kramer MR, Pastan S, Lim SS, McClellan WM. Sociodemographic and geographic predictors of quality of care in United States patients with end-stage renal disease due to lupus nephritis. Arthritis Rheumatol. 2015:67(3):761-72.

12. Plantinga LC, Patzer RE, Drenkard C, Pastan SO, Cobb J, McClellan W, Lim SS. Comparison of quality-of-care measures in U.S. patients with end-stage renal disease secondary to lupus nephritis vs. other causes. BMC Nephrol. 2015;16:39.

13. O'Shaughnessy MM, Montez-Rath ME, Zheng Y, Lafayette RA, Winkelmayer WC. Differences in initial hemodialysis vascular access use among glomerulonephritis subtypes in the United States. Am J Kidney Dis. 2016:67(4):638-47.

14. United States Renal Data System. 2015 USRDS annual data report: Epidemiology of kidney disease in the United States. Bethesda: National Institutes of Health, National Institute of Diabetes and Digestive and Kidney Diseases; 2015.

15. Sule SD, Fadrowski JJ, Fivush BA, Gorman G, Furth SL. Reduced albumin levels and utilization of arteriovenous access in pediatric patients with systemic lupus erythematosus (SLE). Pediatr Nephrol. 2007;22(12):2041-6.

16. Sule SD, Fadrowski JJ, Fivush BA, Neu AM, Furth SL. Persistent low albumin and temporary vascular access in pediatric patients with SLE on hemodialysis. Pediatr Nephrol. 2009;24(10):1981-7.

17. Plantinga LC, Patzer RE, Drenkard C, Kramer MR, Klein M, Lim SS, McClellan WM, Pastan SO. Association of time to kidney transplantation with graft failure among US patients with end-stage renal disease due to lupus nephritis. Arthritis Care Res. 2015:67(4):571-81.

18. Mohan S, Huff E, Wish J, Lilly M, Chen SC, McClellan WM. Recovery of renal function among ESRD patients in the US Medicare program. PLoS One. 2013:8(12):e83447.

19. Sato Y, Miyamoto M, Sueki S, Sakurada T, Kimura K, Nakazawa R, Yoshioka M, Sasaki H, Miyano S, Chikaraishi T. Risk factors associated with inadequate veins for placement of arteriovenous fistulas for hemodialysis. J Artif Organs. 2013; 16(4):469-74.

20. Muringai T, Noble H, McGowan A, Channey M. Dialysis access and the impact on body image: role of the nephrology nurse. Br J Nursing. 2008;17(6):362-6.

21. Moroni G, Tantardini F, Ponticelli C. Renal replacement therapy in lupus nephritis. J Nephrol. 2003:16(6):787-91.

22. Perera GB, Mueller MP, Kubaska SM, Wilson SE, Lawrence PF, Fujitani RM Superiority of autogenous arteriovenous hemodialysis access: maintenance of function with fewer secondary interventions. Ann Vasc Surg. 2004;18(1):66-73.

23. Huber TS, Carter JW, Carter RL, Seeger JM. Patency of autogenous and polytetrafluoroethylene upper extremity arteriovenous hemodialysis accesses: a systematic review. J Vasc Surg. 2003;38(5):1005-11.

24. Saran R, Elder SJ, Goodkin DA, Akiba T, Ethier J, Rayner HC, Saito A, Young EW, Gillespie BW, Merion RM et al. Enhanced training in vascular access creation predicts arteriovenous fistula placement and patency in hemodialysis patients: results from the Dialysis Outcomes and Practice Patterns Study. Ann Surg. 2008:247(5):885-91.

25. Layton JB, Hogan SL, Jennette CE, Kenderes B, Krisher J, Jennette JC, McClellan WM. Discrepancy between Medical Evidence Form 2728 and renal biopsy for glomerular diseases. Clin J Am Soc Nephrol. 2010;5(11):2046-52

26. Plantinga LC, Drenkard C, Pastan SO, Lim SS. Attribution of cause of end-stage renal disease among systemic lupus erythematosus patients: the Georgia Lupus Registry. Lupus Sci Med. 2016:3(1):e000132. 